\title{
Cost-effectiveness of quadrivalent versus trivalent inactivated influenza vaccines for the Portuguese elderly population
}

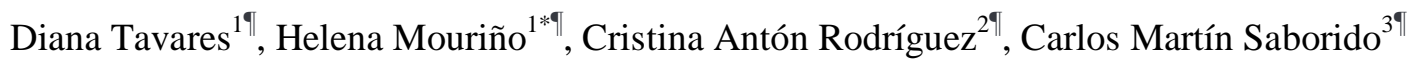

${ }^{1}$ Faculdade de Ciências, Universidade de Lisboa, Lisboa, Portugal

${ }^{2}$ Facultad de Medicina, Universidad Francisco de Vitoria, Madrid, España

${ }^{3}$ Hospital La Paz Institute for Health Research, IP Research on Evidence and Decision Making Group, Higher Education Center Hygiea. UDIMAMadrid, Madrid. España

* Corresponding author

E-mail: mhnunes@fc.ul.pt

II This authors contributed equally to this work.

\section{Abstract}

\section{Background}

Quadrivalent Inactivated Vaccine (QIV) is expected to replace Trivalent Inactivated Vaccine (TIV) over time. In Portugal, TIV is free of charge for risk groups, which include older adults. In its turn, QIV which provides broader protection as it includes an additional lineage B strain - was introduced in Portugal in October 2018, but only from the influenza season 2019/20 was provided free of charge for the risk groups. This study evaluates the cost-effectiveness of switching from TIV to QIV from the National Health Service perspective in the Portuguese elderly mainland population ( $\geq 65$ years old).

\section{Methods}


A decision tree model was developed to compare TIV and QIV, based on Portuguese hospitalization data for the 2015/16 influenza season. The primary health economic outcome under consideration was the Incremental Cost-Effectiveness Ratio (ICER). In addition, a one-Way Sensitivity Analysis was performed to evaluate the impact of model parameters on the ICER; Probabilistic Sensitivity Analysis was also carried out to analyze the robustness of the base case results.

\section{Results}

The high cost of QIV (approximately three times the cost of TIV) would lead to a total increment of $5,283,047 €$, and the resulting ICER would be 26,403,007€/QALY, mainly above the usual willingness-topay threshold.

\section{Conclusions}

From the National Health Service perspective, our findings reveal that QIV is not cost-effective for the Portuguese elderly population due to the high cost. If the QIV costs were the same as the TIV, then QIV would be cost-effective.

Keywords: Influenza vaccines; Decision tree; Cost-Effectiveness analysis; One-way sensitivity analysis; Probabilistic sensitivity analysis

\section{Introduction}

2 Seasonal influenza is an acute respiratory disease caused by infection with influenza viruses(1). There are

3 four types of seasonal influenza viruses: A, B, C and D(1). Only influenza A and B viruses circulate and

4 cause seasonal epidemics of disease(1). The infection may cause signs and symptoms like fever, cough,

5 headache, muscle and joint pain and weakness(1). 
Influenza outbreaks are recorded every year. In temperate zones of the northern and southern

8 In Portugal, influenza outbreaks are characterized by significant morbidity in the general population and

9 increased mortality rates. The high-risk groups are: elderly ( $\geq 65$ years old), patients with chronic or

10 immunosuppressive conditions aged six months or older, pregnant women, health professionals and other

11 healthcare givers(4).

12 An influenza pandemic can appear when a new and different type of influenza A virus emerges, and it

13 can infect humans who are not immunized(1,5). The most recent pandemic was in 2009 , caused by the

$14 \mathrm{~A}(\mathrm{H} 1 \mathrm{~N} 1) 2009$ virus, after which it became a seasonal influenza serotype(1). In Portugal, 1,189 people

15 were hospitalized, around $10 \%$ in intensive care units, resulting in 124 deaths(6).

According to the World Health Organization (WHO), vaccination is the most effective way to prevent seasonal influenza and subsequent severe outcomes(1). Therefore, vaccination should be administered annually to provide optimal protection. Influenza Surveillance System in Portugal is usually activated on week 40 (October) of a given year $(n)$ and lasts up to week 20 (April) of the following year $(n+1)(7,8)$.

27 vaccination coverage was $50.1 \%$ in Portugal(7). The coverage rate has still increased in the last few years.

28 For instance, in the 2017/18 season, it has increased to $60.8 \%$ for people aged 65 years or above(11). 
31 groups ( $\geq 65$ years old) during a single season(12). In the European Union, it is estimated that influenza

32 vaccination prevents up to 37,000 deaths each year(13).

33 There are two main types of seasonal influenza vaccines, namely Inactivated Influenza Vaccine (IIV)

34 and Lived Attenuated Influenza Vaccine (this one is not within the scope of the paper) $(5,12)$. The different

35 IIV developed are: trivalent, trivalent adjuvanted and quadrivalent. Trivalent Inactivated Vaccine (TIV) is

36 the most common IIV and protect against three different influenza viruses (two influenza A strains and

37 one influenza B lineage). In some EU/EEA countries, the trivalent adjuvanted vaccine is available for

38 older people to empower immune response (5,14). Quadrivalent inactivated Influenza Vaccine (QIV) is

39 available since the 2014/2015 season in some EU/EEA countries, and it is expected to replace TIV over

40 time(5). It contains one more influenza B strain in addition to those included in TIV, which provides wider

41 protection against influenza viruses. TIV is available in all EU/EEA countries, while QIV is only available

42 in some of them. In Portugal, QIV was launched in October 2018, but only from the influenza season

$432019 / 20$ it was provided free of charge for the risk groups.

44 Influenza is, thus, a major global health threat that affects all countries, not only from a health

45 perspective but also from economic and social approaches. Therefore, it is crucial to carry out economic

46 evaluations of the influenza vaccination programmes.

47 This work aims to estimate the cost-effectiveness of switching from TIV to QIV based on the National

48 Health Service perspective. The results are presented as incremental cost per unit of effect, described by

49 the Incremental Cost-Effectiveness Ratio (ICER). As far as we know, no cost-effectiveness analysis of

50 Portuguese influenza vaccination has been reported in the literature yet.

51

52 Methods 


\section{Key Features of the Economic Evaluation}

54 The target population is the population of mainland Portugal aged 65 and above because they are one of

55 the most relevant influenza risk groups. In 2015, they represented 21\% of the Portuguese population(15).

56 This group is usually retired, so productivity losses are not relevant. The cost-effectiveness evaluation was

57 performed from the National Health Service (NHS) perspective, where only direct costs were considered.

58 Thus, only the medical costs paid by the NHS are considered here.

The Influenza Surveillance System runs from week 40 of a specific year until week 20 of the following year, so the time horizon established for this work was one year. Therefore, data under analysis refers to the $2015 / 16$ influenza season.

As stated above, economic evaluations are performed to identify, measure, value and compare the costs and consequences of different therapeutic alternatives in what respects to influenza vaccination. To perform the cost-effectiveness analysis, health outcomes generated by the two alternative interventions under analysis are assessed through the Quality-Adjusted Life Year (QALY). It measures the length of life-years (i.e., quantity gains) and the quality of life (i.e., quality gains). The quality of life is quantified by the notion of utility: the greater the preference for a particular state, the greater its utility. Utilities incorporate in the analysis the preferences of individuals for different treatment-related outcomes. Another key measure is the concept of quality-of-life loss due to the disease (influenza, in this case), that is, disutility. It corresponds to the reverse of utility, i.e., decrease in utility due to serious health complications. These quantities are obtained by Health-related quality of life (HRQoL) instruments, such

72 as the EQ-5D family of questionnaires. Studies about utilities and disutilities for the Portuguese

73 population are very scarce. In this work, utilities associated with different health states were obtained from

74 a study on EQ-5D Portuguese Population norms(16). Data available were stratified by age, but

75 unfortunately, there was no information for people aged 65 or over. Therefore, the utilities for that age

76 group were derived by interpolation (see S1 Appendix). Moreover, no Portuguese studies on disutilities 
77 were found for influenza. Such data were extracted from a Spanish study (17) (see S1 Appendix). All

78 these results were thus combined in a single index, QALY $(18,19)$.

79 Incremental Cost-Effectiveness Ratio (ICER) is the primary cost-effectiveness outcome used in this

80 work(19). To make recommendations to policymakers, ICER is usually compared with an established

81 threshold, called Willingness-To-Pay (WTP). Some decision-makers have established a Willingness-To-

82 Pay (WTP) threshold, that refers to the maximum ICER accepted for an intervention to be considered

83 cost-effective. In Portugal, there is no established cost-effectiveness threshold for health interventions.

84 Some Portuguese authors refer to a WTP threshold of 30,000€/QALY(20,21), while others consider the

85 cost-effectiveness threshold twice the Gross Domestic Product (GDP) per capita or Gross National Income

86 (GNI) per capita(21,22). In 2015, the Portuguese GNI per capita was 16,887.36€, resulting in a ceiling

87 ratio of approximately $34,000 €$ (twice the GNI)(23,24). In this paper, the ICER will be used to provide

88 information on the extra amount that is necessary to pay in order to gain an extra QALY when the most

89 effective alternative is chosen(19,25).

\section{Decision Tree}

91 The cost-effectiveness analysis was based on a decision tree model, a very powerful tool in this context

92 because it incorporates probabilities of the outcomes, resource costs, vaccination coverage rate, prices of

93 the vaccines, efficacy, utilities and disutilities. It consists of a series of pathways representing possible

94 prognoses for each alternative therapy being evaluated. A static model was used, and no herd effects of

95 vaccination were evaluated.

96 One decision tree model was developed for each alternative therapy (TIV and QIV). For convenience,

97 each decision tree was split into two different diagrams referring to vaccinated and unvaccinated people.

98 For illustration, Fig 1 displays the branch of the decision tree for vaccinated individuals. 
99 Fig 1. Decision tree for the cost-effectiveness analysis. Branch for the vaccinated people aged $\geq 65$

100 years old.

101 ILI, influenza-like-illness; H, healthy; D, death; Conf. Influenza, Confirmed Influenza; GP Consultation,

102 General Practitioner Consultation; Pneu. Hosp., Hospitalization due to pneumonia; RD Hosp.,

103 Hospitalization due to respiratory disease; HD Hosp., Hospitalization due to heart disease; Inf. Hosp.,

104 Hospitalization due to Influenza.

105 It was assumed that influenza confirmation (through diagnostic tests) was carried out in the context of

106 General Practitioner (GP) consultation, pneumonia hospitalization, respiratory disease hospitalization or

107 heart disease hospitalization. Although it is known that influenza may be diagnosed in many other

108 situations, the diseases described above are usually associated with complications due to influenza in the

109 elderly. It should be noted that influenza event (as a secondary diagnosis) may have occurred before or

110 after hospital admission, in which principal diagnoses was pneumonia, respiratory disease or heart disease.

111 It is important to note that the "Healthy" state is assumed to include all individuals who did not die,

112 regardless of their health conditions.

113 Input Parameters and Computation of the Probabilities

114 The decision tree is powered by different types of parameters. A detailed description of each of these

115 measures is provided in the S2 Appendix (which includes S1 Table). A summary of the base case values

116 for the parameters under consideration is displayed in Table 1. The computation of the different

117 parameters involved in the decision tree pathways for the TIV - probabilities, costs and QALY - are given

118 in the S1 Fig and S2 Table. Similar calculations are made for the QIV scenario. 
121 Table 1. Input parameters for the decision tree. Base case values.

\section{Input Parameters}

Population

Vaccination Coverage Rate

Trivalent Vaccine Effectiveness

Quadrivalent Vaccine Effectiveness

Probability of Influenza-like-illness

Probability of Confirmed Influenza

Probability of GP Consultation

Probability of Hospitalization due to Influenza

Probability of death when Hosp. Influenza

Probability of Hospitalization due to Pneumonia

Probability of death due to Hosp. Pneumonia

Probability of Hospitalization due to Respiratory Disease

Probability of death when Hosp. RD

Probability of Hospitalization due to Heart Disease

Probability of death when Hosp. HD

Probability of death when no confirmed influenza

Cost of GP consultation

Cost of Hospitalization due to Influenza

Cost of death when Hospitalization due to Influenza

Cost of Hospitalization due to Pneumonia

Cost of death when Hospitalization due to Pneumonia

Cost of Hospitalization due to Respiratory Disease

Cost of death when Hospitalization due to RD

Cost of Hospitalization due to Heart Disease

Cost of death when Hospitalization due to HD

Cost of antiviral treatment

Cost of trivalent vaccine

\section{Base Case Source}

$2,067,654$

(26)

0.501

0.580

0.599

(27-35)

0.007

$(7,12,26,31,3$

0.278

0.952

0.091

0.079

0.008

0.381

0.014

0.050

0.026

0.110

0.043

31.000

2644.011

6364.597

2906.749

11298.219

2534.841

1363.460

2978.842

7785.951

18.300

2.576 
Cost of quadrivalent vaccine

Cost of vaccine administration

Disutility associated with ILI without influenza confirmation

Disutility associated with no hospitalized influenza

Disutility associated with hospitalization due to influenza

Disutility associated with hospitalization due to pneumonia

Disutility associated with hospitalization due to respiratory disease

Disutility associated with hospitalization due to heart disease

Utility associated with healthy population
7.692

3.700

0.009

0.009

0.031

0.031

0.031

0.031

0.625
$(43,44)$

\section{Scenario Analysis}

124 The most common ways to characterize parameter uncertainty are: One-way Sensitivity Analysis (OWSA) 125 and Probabilistic Sensitivity Analysis (PSA)(19,25).

OWSA consists of varying the point estimates of the input parameters one at a time within a plausible range of values and assessing the impact on model outcomes $(19,25)$, which corresponds to the ICER. When the Confidence Interval (CI) for a specific parameter was known from the literature, these limits were used as the OWSA range. For parameters with no CI available, a range of $\pm 20 \%$ was used to assess model's sensitivity to parameters variation. A tornado diagram was used to report OWSA results.

A scenario analysis of the vaccination coverage rate, TIV effectiveness and QIV effectiveness variation was also carried out. The cost of the quadrivalent vaccine was also studied in detail, as it was identified as a critical parameter(45). For vaccination coverage rate and QIV cost, a sequence of parameters values was generated with an increment of 0.05. For TIV and QIV effectiveness, the increment used was 0.005. 
137 values used for each input parameter of the decision tree models. It also shows the probability distribution

138 (with information on location, shape, scale, when appropriate) used for each parameter under

139 consideration. A total of 1,000 Monte Carlo (MC) simulations were performed.

\section{Results}

\section{Base Case Analysis}

142 Table 2 summarises the base case results of shifting from TIV to QIV in the elderly, based on the 2015/16

143 influenza season data. Results show a difference in confirmed influenza cases of approximately 37 . As a

144 result, about 36 GP consultations could have been averted and, therefore, 1,103€ saved. In addition, five

145 hospitalizations, including hospitalizations due to influenza, pneumonia, respiratory disease and heart

146 disease, could have been averted and one life saved.

147 Table 2. Base Case. Results of the cost-effectiveness evaluation comparing TIV and QIV.

\begin{tabular}{lccc}
\hline & TIV & QIV & $\begin{array}{c}\text { Difference } \\
\text { (QIV-TIV) }\end{array}$ \\
\hline Events & 2631.35 & 2595.77 & -35.58 \\
GP Consultations & 239.00 & 235.77 & -3.23 \\
Hospitalizations due to Influenza & 19.00 & 18.74 & -0.26 \\
Deaths due to Influenza Hospitalization & 21.00 & 20.72 & -0.28 \\
Hospitalizations due to Pneumonia & 8.00 & 7.89 & -0.11 \\
Deaths due to Pneumonia Hospitalization & 40.00 & 39.46 & -0.54 \\
Hospitalizations due to RD & 2.00 & 1.97 & -0.03 \\
Deaths due to RD Hospitalization & 73.00 & 72.01 & -0.99 \\
Hospitalizations due to HD & 8.00 & 7.89 & -0.11 \\
Deaths due to HD Hospitalization & $1,035,895$ & $1,035,895$ & 0.00 \\
Vaccine Doses & & &
\end{tabular}

Costs

GP Consultations $\quad 81,572 € \quad 80,469 € \quad-1,103 €$ 


Hospitalizations due to Influenza
Deaths due to Influenza Hospitalization
Hospitalizations due to Pneumonia
Deaths due to Pneumonia Hospitalization
Hospitalizations due to RD
Deaths due to RD Hospitalization
Hospitalizations due to HD
Deaths due to HD Hospitalization
Vaccine Doses
Total

\section{QALYs}

Total

$\begin{array}{ccc}710,464 € & 701,189 € & -9,275 € \\ 121,552 € & 119,934 € & -1,617 € \\ 128,213 € & 126,508 € & -1,705 € \\ 90,401 € & 89,189 € & -1,211 € \\ 99,125 € & 97,841 € & -1,285 € \\ 2,731 € & 2,697 € & -34 € \\ 256,048 € & 252,687 € & -3,361 € \\ 62,303 € & 61,471 € & -831 € \\ 2,668,465 € & 7,968,557 € & 5,300,092 € \\ 8,018,570 € & 13,301,618 € & 5,283,047 €\end{array}$

$1265456.95 \quad 1265457.15$

0.20

$\operatorname{ICER}(€ / Q A L Y)$

$26,403,007$

Regarding hospitalization and death costs, caution must be taken in the interpretation of the results.

150 The cost of death, when hospitalized, includes all hospitalization costs, and in the same way, the

151 hospitalization costs also include the costs of people who died. Thus, $15,625 €$ could have been saved in

152 hospitalizations: $9,275 €$ in hospitalizations due to influenza; $1,705 €$ in hospitalizations due to

153 pneumonia; $1,285 €$ in hospitalizations due to respiratory disease; and 3,361 € in hospitalizations due to

154 heart disease. From that value, 3,694€ are related to hospitalizations of patients who died (Table 2).

155 In what concerns the number of vaccinated people (mentioned in Table 2 as vaccine doses), the value

156 is the same for both strategies. It was an expected result because the vaccination coverage rate applied to

157 TIV and QIV models was the same (the paper's focus is to evaluate the cost-effectiveness of shifting from

158 TIV to QIV). However, there is a difference in the expected costs computed from the two strategies

159 because they depend on the cost of each vaccine. A total of 1,035,895 vaccine doses were expected to

160 have been administered to individuals aged $\geq 65$ years old in the $2015 / 16$ season. For the TIV strategy, this 
QIV would result in an additional cost of 5,300,092 € (Table 2).

The expected difference in costs between TIV and QIV is 5,283,047 €, while the difference in effects

164 (QALYs) is expected to be 0.20. These values result in a base case ICER of 26,403,007€/QALY (Table

2). The plot of the differences in costs and effects on the cost-effectiveness plane shows the value under

GNI per capita), the new vaccine would not be cost-effective.

\section{One-way Sensitivity Analysis}

Fig 2 summarizes the one-way sensitivity analysis using the tornado diagram.

Fig 2. One-way sensitivity analysis. Tornado diagram.

Fig 2 reveals that the disutility associated with ILI when no confirmed influenza, and the disutility associated with no hospitalized influenza have the highest impact on the ICER. For example, if the first parameter mentioned above assumes the value of 0.007 (lower bound), it results in an ICER of 19,219,556 €/QALY; and the value of 0.011 (upper bound) results in an ICER of 42,160,989 €/QALY. This can be explained by the increase in incremental QALYs, yielded by decreasing this parameter, resulting in a reduced ICER and vice-versa. In contrast, the variation of the second parameter within the 95\% CI (0.007, 0.011) produced ICERs of 39,019,821€/QALY and 19,951,738€/QALY, respectively. Thus, while the pathway corresponding to vaccinated patients with ILI but not confirmed influenza is more populated in the QIV model than in TIV, the same is not valid for the pathway referring to vaccinated patients with no hospitalized influenza. Thus, a reduction in disutility associated with no hospitalized influenza would 182 decrease incremental QALYs and increase ICER. 
The cost of the quadrivalent vaccine is the third parameter appearing in the tornado diagram (Fig 2).

The variation of the cost of the quadrivalent vaccine within a $\pm 20 \%$ range results in an ICER of 18,438,140 €/QALY (lower bound) and 34,367,874€/QALY (upper bound), respectively. The high impact of QIV's cost on ICER was expected if attention is paid to the difference in costs of QIV and TIV vaccine doses (Table 2). The cost of QIV's vaccines doses is largely above the cost of TIV's vaccine doses.

Further analysis was performed, varying the QIV cost within a broader range (Fig 3). For a QIV cost equal to TIV cost, an ICER of $-85,182 € /$ QALY was obtained, meaning that shifting from TIV to QIV would be cost-saving, as expected. However, a value higher than $2.576 €$ would quickly lead to being not costeffective.

Fig 3. One-way sensitivity analysis of the cost of QIV. Variation between $2.576 €$ (the cost of TIV) and

$9.756 €$ with increments of $0.05 €$.

The probability of ILI and the probability of confirmed influenza also have a high impact on ICER (Fig

2). Varying these parameters $20 \%$ downward and upward produced ICERs of 33,025,054€/QALY and $21,988,309 € / Q A L Y$, respectively. Thus, as the probability of ILI and/or the probability of confirmed influenza increases, the ICER decreases.

One-way sensitivity analyses of the effectiveness of QIV and TIV are displayed in S2 Fig and S3 Fig, respectively. If the TIV effectiveness is equal to QIV effectiveness, the ICER is not defined as it results in a difference in effects equal to zero and, therefore, a division by zero, and a vertical asymptote at $59.91 \%$

202 is shown (S2 Fig). As TIV effectiveness approaches $59.91 \%$ from the left, the ICER tends to positive

203 infinity. Otherwise, as TIV effectiveness approaches $59.91 \%$ from the right, the ICER tends to negative

204 infinity. Such behavior is explained by the difference in effects close to zero when TIV and QIV

205 effectiveness are close. A negative ICER is produced for TIV effectiveness values higher than QIV

206 effectiveness as the incremental QALYs value is negative. Similarly, S3 Fig shows a vertical asymptote 
when QIV effectiveness equals 58\%; ICER tends to infinity when it approaches the vertical asymptote

Although vaccination coverage rate does not significantly impact ICER as the above parameters do, a detailed study was also performed for this parameter (S4 Fig). A higher coverage rate would result in

\section{Probabilistic Sensitivity Analysis}

214 Probabilistic sensitivity analysis allows one to evaluate the robustness of the base case results. To attain

215 this goal, a probability distribution was assigned to each parameter of the model to reflect parameter

216 uncertainty due to sampling errors. Parameters values were then sampled 1,000 times; expected costs and

217 QALY were recorded from the 1,000 MC simulations. Finally, some empirical statistics such as mean,

218 standard deviation and confidence intervals were computed.

In general, the results from PSA (S3 Table) are similar to those obtained from the base case analysis

(Table 2). The estimated mean costs difference between QIV and TIV is 5,293,456 € with an interval

224 the base case result.

S5 Fig displays the scatterplot of the 1,000 MC simulations to compare TIV and QIV, that is, the 
Fig 4 shows the results of the simulations in a cost-effectiveness plane, where the incremental costs are

231 plotted against the incremental effects. According to Fig 4, the cost-effectiveness results are robustly

232 located in the first quadrant of the cost-effectiveness plane, and all simulated ICERs are higher than CE

233 thresholds previously established.

234 Fig 4. Probabilistic sensitivity analysis for TIV and QIV. Cost-effectiveness plane based on data from 235 the 2015/16 influenza season.

\section{Discussion}

237 To the best of our knowledge, this is one of the first published studies performing a cost-effectiveness

238 evaluation of seasonal influenza vaccination in Portugal. Only a few papers studied the burden of disease

239 and vaccination effectiveness in the Portuguese population, for instance, the international I-MOVE+

240 project(46-50).

We evaluated the cost-effectiveness of switching from TIV to QIV based on the 2015/16 influenza season data. Base case results revealed that the universal substitution of TIV with QIV would result in an ICER of 26,403,007€ per QALY gained. As such, for the cost-effectiveness thresholds of 30,000€/QALY or 34,000€/QALY, QIV is not cost-effective. Probabilistic sensitivity analysis enhanced the robustness of

245 the base case results. The ICER is much higher than any possible ceiling ratio established by NHS. Such

246 results are comparable to those obtained for the first year of QIV administration to Hong Kong elderly

247 ( $\geq 80$ years old) when a cost-effectiveness analysis over nine seasons was performed(51). The need for a

248 longer time horizon study is here emphasized.

The majority of the published international papers(45,52-60)does not report such a high ICER, and

250 QIV is usually identified as cost-effective compared with TIV. As cited above, one of the reasons might

251 be using of a long time horizon to calculate QIV effectiveness, which allowed to assume a few seasons

252 that match the TIV with the circulating strains and therefore low mismatching (see S2 Appendix). Another 
reason might be the cost of QIV, which in this study is about three times the TIV. Regarding the

254 quadrivalent vaccine, its cost is here $200 \%$ higher than TIV. However, in the literature, the additional cost

255 of QIV is rarely higher than $100 \%$ of the TIV cost(52,54-56), which might be one of the reasons why QIV

256 is cost-effective in some studies. To be cost-effective, the cost of QIV would need to be the same as TIV

257 in our case.

258 In the literature, the probability of influenza-like-illness and the probability of confirmed influenza was

259 found to be higher than those used here. For example, Capri et al.(60) applied a probability of influenza of

$2606.4 \%$, resulting from the product of ILI attack rate $(16.8 \%)$ by influenza virus isolation rate $(32.1 \%)$, while

261 Van Bellinghen et al.(55) used a probability of symptomatic influenza infection of $6.17 \%$. In our study,

262 the probability of confirmed influenza was derived from a Portuguese report on influenza surveillance,

263 and the probability of ILI was determined from other input values. It is comparable to the value reported

264 by INSA for the 2015/16 influenza season(31).

One-way sensitivity analysis demonstrated that our model is more sensitive to disutility associated with

ILI, disutility associated with no hospitalized influenza, cost of quadrivalent vaccine and probabilities of

base case conclusions and confirm their robustness.

A limitation of our study has to do with the fact that the model is static in a way that herd effects of immunization are not considered, and only direct protection is captured. Thus, the impact of vaccination on the burden of the disease might be underestimated. In the future, a dynamic transmission model might

273 be a possible approach to account for the impact of vaccination on influenza transmission $(45,56)$. In

274 addition, side effects of vaccination were not included in this study, but QIV safety is assumed to be 275 comparable to that of TIV, and side effects are assumed to be mild and temporary. Thus, no impact is 276 expected on the $\operatorname{ICER}(56)$. 
277 Summing up, this study contributes to understanding the impact of annual influenza epidemics on

278 health economics and public health in Portugal.

\section{Acknowledgments}

280 H. Mouriño was supported by the National Funding from FCT - Fundação para a Ciência e Tecnologia,

281 under the project: UIDB/04561/2020.

\section{References}

283 1. WHO | Influenza (Seasonal). WHO [Internet]. 2018; Available from:

284 http://www.who.int/mediacentre/factsheets/fs211/en/

285 2. Shi A, Zeng D, Lin S-S, Caldwell L. Harrison's - Principles of Internal medicine. 16th ed.

286 McGraw-Hill; 2011. 515 p.

287 3. Villacampa T. Manual Curso Intensivo Mir Asturias - Enfermedades Infecciosas. 2017.

288 4. Direção Geral de Saúde. Orientação Vacinação contra a gripe. Época 2017/2018. 2017;1-6.

289 5. Types of Influenza Viruses | Seasonal Influenza (Flu) | CDC [Internet]. [cited 2018 Mar 6].

290 Available from: https://www.cdc.gov/flu/about/viruses/types.htm

291 6. George F. História da gripe. 2014;1-28.

292 7. Sousa Uva M, Kislaya I, Roquette R, Rodrigues AP, Machado A. Vacinação antigripal da

293 população portuguesa na época 2015/2016: estudo na amostra ECOS. 2016 Oct 13 [cited 2018 Sep

294 1]; Available from: http://repositorio.insa.pt/handle/10400.18/4013

295 8. Instituto Nacional de Saúde Dr. Ricardo Jorge. Influenza Vaccine Effectiveness in Portugal.

296 Season 2015-16 Report. 2017. 44 p. 
297 9. Key Facts About Influenza (Flu) | Seasonal Influenza (Flu) |CDC [Internet]. [cited 2018 Mar 9]. Available from: https://www.cdc.gov/flu/keyfacts.htm

10. Jorgensen P, Mereckiene J, Cotter S, Johansen K, Tsolova S, Brown C. How close are countries of the WHO European Region to achieving the goal of vaccinating $75 \%$ of key risk groups against

11. Machado A, Torres AR, Kislaya I, Neto M. Vacinação antigripal da população portuguesa em influenza? Results from national surveys on seasonal influenza vaccination programmes, 2008/2009 to 2014/2015. Vaccine [Internet]. 2017;36(4):442-52. Available from: https://doi.org/10.1016/j.vaccine.2017.12.019

12. Demicheli V, Jefferson T, C DP, Ferroni E, Thorning S, Re T, et al. Vaccines for preventing influenza in the elderly ( Review ). 2018;(2).

13. Influenza - European Commission [Internet]. [cited 2018 Mar 15]. Available from: https://ec.europa.eu/health/vaccination/influenza_en

14. Types of seasonal influenza vaccine |WHO. 2018 Mar 22 [cited 2018 Mar 22]; Available from: http://www.euro.who.int/en/health-topics/communicable-diseases/influenza/vaccination/types-of-

15. Krahn M, Kuntz KM, Meltzer DO, Owens DK, Russell LB, Siegel JE, et al. Recommendations for on Cost-Effectiveness in Health and Medicine. JAMA. 2016;316(10):1093-103. Res. 2014;23(2):425-30. 
17. Hollmann M, Garin O, Galante M, Ferrer M, Dominguez A, Alonso J. Impact of Influenza on Health-Related Quality of Life among Confirmed (H1N1)2009 Patients. PLoS One. 2013;8(3):110.

18. Greenland S. Sensitivity analysis, Monte Carlo risk analysis, and Bayesian uncertainty assessment. Risk Anal [Internet]. 2001;21(4):579-83. Available from: http://www.ncbi.nlm.nih.gov/pubmed/11726013

19. Drummond MF, Sculpher MJ, Claxton K, Stoddart GL, Torrance GW. Methods for the Economic Evaluation of Health Care Programmes. Fourth. Oxford; 2015.

20. Laires PA, Ejzykowicz F, Hsu T-Y, Ambegaonkar B, Davies G. Cost-effectiveness of adding ezetimibe to atorvastatin vs switching to rosuvastatin therapy in Portugal. J Med Econ [Internet]. 2015;18(8):565-72. Available from:

21. Cameron D, Ubels J, Norström F. On what basis are medical cost-effectiveness thresholds set? http://www.tandfonline.com/doi/full/10.3111/13696998.2015.1031794

23. Areia M, Spaander MCW, Kuipers EJ, Dinis-Ribeiro M. Endoscopic screening for gastric cancer: A cost-utility analysis for countries with an intermediate gastric cancer risk. United Eur

24. World Development Indicators | DataBank [Internet]. [cited 2018 Sep 15]. Available from: http://databank.worldbank.org/data/reports.aspx?source=2\&country=PRT 
25. Briggs A, Sculpher M, Claxton K. Decision Modelling for Health Economic Evaluation. Oxford: Oxford University Press; 2006. 445 p.

26. Portal do Instituto Nacional de Estatística [Internet]. [cited 2018 May 4]. Available from: https://www.ine.pt/xportal/xmain?xpid=INE\&xpgid=ine_indicadores\&indOcorrCod=0000256\&co ntexto $=$ bd\&selTab=tab2\&xlang=en

27. Guiomar R, Conde P, Cristóvão P, Pechirra P, Nunes B. Programa Nacional de Vigilância da Gripe - Relatório da Época 2012/2013. Inst Nac Saúde Doutor Ricardo Jorge, IP. 2013;

28. Guiomar R, Conde P, Cristóvão P, Pechirra P, Nunes B. Programa Nacional de Vigilância da Gripe - Relatório da Época 2013/2014. Inst Nac Saúde Doutor Ricardo Jorge, IP. 2015; Vigilância da Gripe - Relatório da época 2014/2015. Inst Nac Saúde Doutor Ricardo Jorge, IP. 2015;

30. Guiomar R, Pechirra P, Cristóvão P, Costa I, Conde P, Rodrigues AP, et al. Programa Nacional de

31. Guiomar R, Pechirra P, Rodrigues AP, Silva SP, Machado A, Nunes B. Programa Nacional de Vigilância da Gripe - Relatório da época 2016/2017. Inst Nac Saúde Doutor Ricardo Jorge, IP. 2017

32. Gonçalves P, Pechirra P, Conde P, Guiomar R, Nunes B. Programa Nacional de Vigilância da Vigilância da Gripe - Relatório da época 2015/2016. Instituto Nacional de Saúde Doutor Ricardo Jorge, editor. Inst Nac Saúde Doutor Ricardo Jorge, IP [Internet]. 2016; Available from: http://repositorio.insa.pt//handle/10400.18/4044 
34. Petri E, Ruiz-aragón J. A Comparison of Cost Effectiveness of Seasonal Influenza vaccines in Spain. In: IVW conference, Albufeira, 6th -9th October 2015. 2015. p. 7015.

35. Instituto Nacional de Saúde Doutor Ricardo Jorge. Boletim de Vigilância Epidemiológica da Gripe - semana 20. 2018.

36. Inverno e Saúde - Evolução Semanal [Internet]. [cited 2018 May 9]. Available from: http://saudesazonal.min-saude.pt/\#consultaSindromeGripal/149/757

37. Administração Central do Sistema de Saúde I.P. Base de dados Central de Morbilidade Hospitalar.

38. Portal do Instituto Nacional de Estatística [Internet]. [cited 2018 May 4]. Available from:

39. Coelho PP, Morgado C, Albuquerque D De, Rodrigues M. Diário da República 1. a série, Nº153 Portaria 234/2015 de 7 de agosto - Anexo-II. 2015.

40. BASE:contratos públicos online [Internet]. [cited 2018 May 4]. Available from: http://www.base.gov.pt/Base/pt/Homepage

41. Direção Geral de Saúde. Orientação 007/2015 Terapêutica e quimioprofilaxia da gripe sazonal. $2014 ; 1-7$.

42. Serviços Partilhados do Ministério da Saúde. Contrato Fornecimento de V13 - Vacina Contra a Gripe Inativada [UDOSE] à Administração Regional de Saúde do Alentejo. 2016.

43. BASE:contratos públicos online [Internet]. [cited 2020 Jan 27]. Available from: http://www.base.gov.pt/Base/pt/Pesquisa/Contrato?a=5864096

44. Portal do INE - Índice de preços no consumidor (Taxa de variação média dos últimos 12 meses Base 2012 - \%) por Localização geográfica e Agregados especiais; Mensal [Internet]. [cited 2020 
Jan 13]. Available from:

https://www.ine.pt/xportal/xmain?xpid=INE\&xpgid=ine_indicadores\&indOcorrCod=0002390\&co ntexto=pi\&selTab=tab0

45. Boer PT, Crépey P, Pitman RJ, Macabeo B, Chit A, Postma MJ. Cost-Effectiveness of Quadrivalent versus Trivalent Influenza Vaccine in the United States. Value Heal [Internet].

46. Rondy M, Kissling E, Gherasim A, Pebody R, Trebbien R, Pozo F, et al. Interim 2017 / 18 influenza seasonal vaccine effectiveness $\square$ : combined results from five European studies. Euro

47. Rondy M, Gherasim A, Casado I, Launay O, Rizzo C, Pitigoi D, et al. Low 2016/17 season vaccine Surveill. 2018;23(9):1-12.

48. Rondy M, Larrauri A, Casado I, Alfonsi V, Pitigoi D, Launay O, et al. 2015 / 16 seasonal vaccine effectiveness against hospitalisation with influenza A ( H1N1) pdm09 and B among elderly people

49. Nunes B, Machado A, Pechirra P, Falcão I, Gonçalves P, Conde P, et al. Efetividade da vacina antigripal na \{é\}poca 2010-2011 em Portugal: resultados do projeto EuroEVA. Rev Port Med

50. Kissling E, Valenciano M, Pozo F, Vilcu A-M, Reuss A, Rizzo C, et al. 2015/16 I-MOVE/IMOVE+ multicentre case control study in Europe: moderate vaccine effectiveness estimates against influenza $\mathrm{A}(\mathrm{H} 1 \mathrm{~N} 1) \mathrm{pdm} 09$ and low estimates against lineage mismatched influenza $\mathrm{B}$ Geral E Fam. 2012;28(4):271-84. among children. Influenza Other Respi Viruses. 2017 Nov 10;

51. You JHS, Ming WK, Chan PKS. Cost-effectiveness analysis of quadrivalent influenza vaccine 
52. You JHS, Ming WK, Chan PKS. Cost-effectiveness of quadrivalent influenza vaccine in Hong Kong - A decision analysis. Hum Vaccines Immunother. 2015;11(3):564-71.

53. Chit A, Roiz J, Aballea S. An assessment of the expected cost-effectiveness of quadrivalent influenza vaccines in Ontario, Canada using a static model. PLoS One [Internet]. 2015;10(7):1-15.

54. Chit A, Roiz J, Briquet B, Greenberg DP. Expected cost effectiveness of high-dose trivalent Available from: http://dx.doi.org/10.1371/journal.pone.0133606 multi-cohort model. PLoS One. 2014;9(6).

56. Dolk C, Eichner M, Welte R, Anastassopoulou A, Van Bellinghen LA, Poulsen Nautrup B, et al. Cost-Utility of Quadrivalent Versus Trivalent Influenza Vaccine in Germany, Using an Individual-

426 57. García A, Ortiz de Lejarazu R, Reina J, Callejo D, Cuervo J, Morano Larragueta R. CostBased Dynamic Transmission Model. Pharmacoeconomics. 2016;34(12):1299-308. effectiveness analysis of quadrivalent influenza vaccine in Spain. Hum Vaccines Immunother. 2016;12(9):2269-77. 
60. Capri S, Barbieri M, de Waure C, Boccalini S, Panatto D. Cost-effectiveness analysis of different seasonal influenza vaccines in the elderly Italian population. Hum Vaccin Immunother. 2018;14(6):1331-41.

61. MC W, G T, Mcguire A. QALYs $\square$ : The Basics. Value Heal. 2009;12(1):S5-9.

62. Uva MS, Kislaya I, Roquette R, Rodrigues AP, Machado A. Vacinação antigripal da população portuguesa na época 2015/2016. Estudo na amostra ECOS. 2016; Available from:

445 Supporting Information

446 S1 Appendix.

447 S2 Appendix. Model input parameters.

448 S1 Table. Proportion of B lineage influenza virus not included in the seasonal trivalent 449 vaccines from $2010 / 11$ to $2017 / 18$ influenza seasons.

450 S2 Table. Description of the variables that take part of S1 Fig.

451 S3 Table. Input parameters. Base case value; SD; parameters of the probability distribution.

452 S4 Table. Probabilistic sensitivity analysis. Assessing the 2015/16 influenza season-related 453 uncertainty. 
454 S1 Fig. Probability, cost and QALY calculations by decision tree pathway for individuals 455 aged $\geq 65$ years old who received TIV.

456 S2 Fig. One-way sensitivity analysis of TIV effectiveness. Variation between $34 \%$ and $74 \%$

457 with increments of 0.005 .

458 S3 Fig. One-way sensitivity analysis of QIV effectiveness. Variation between 58\% (TIV

459 effectiveness) and $100 \%$ with increments of 0.005 .

460 S4 Fig. One-way sensitivity analysis of vaccination coverage rate. Variation between $0 \%$ and $461 \quad 100 \%$ with increments of 0.05 .

462 S5 Fig. Probabilistic sensitivity analysis for TIV and QIV. Scatter plot based on the 2015/16 463 influenza season. 


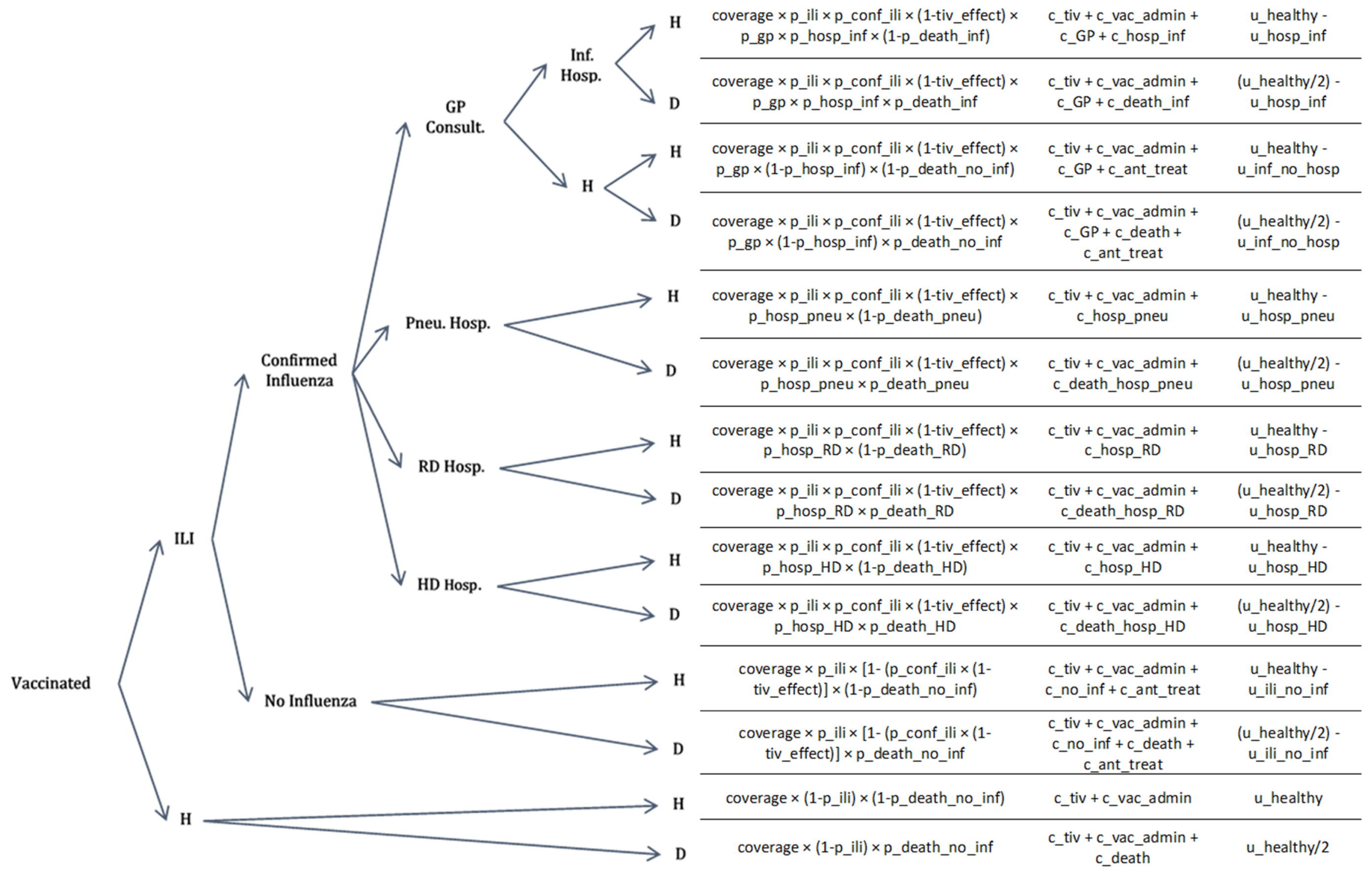




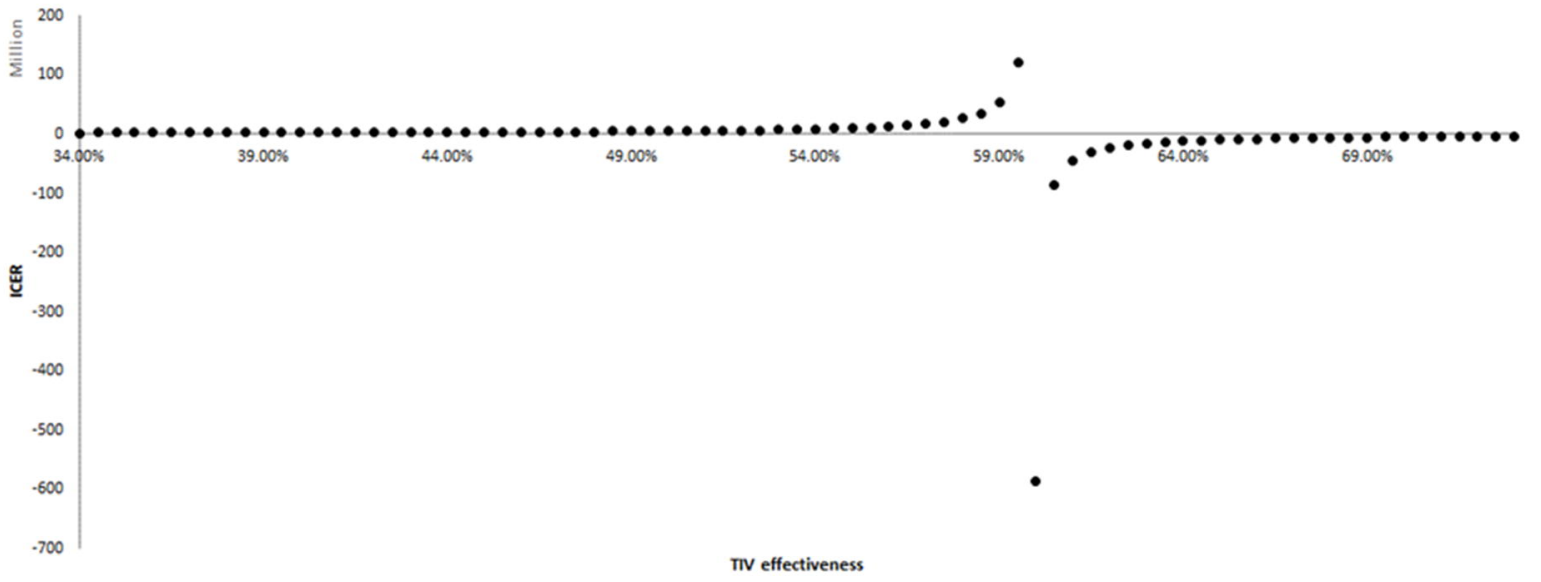




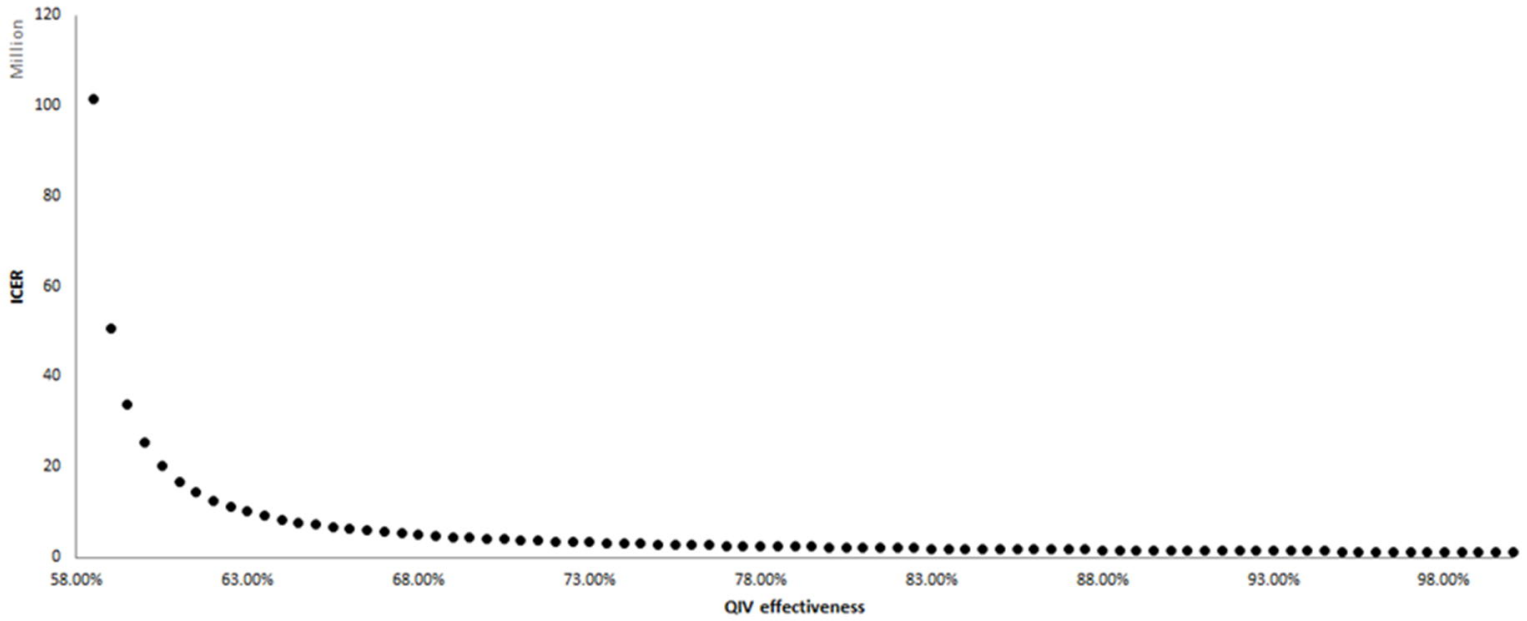




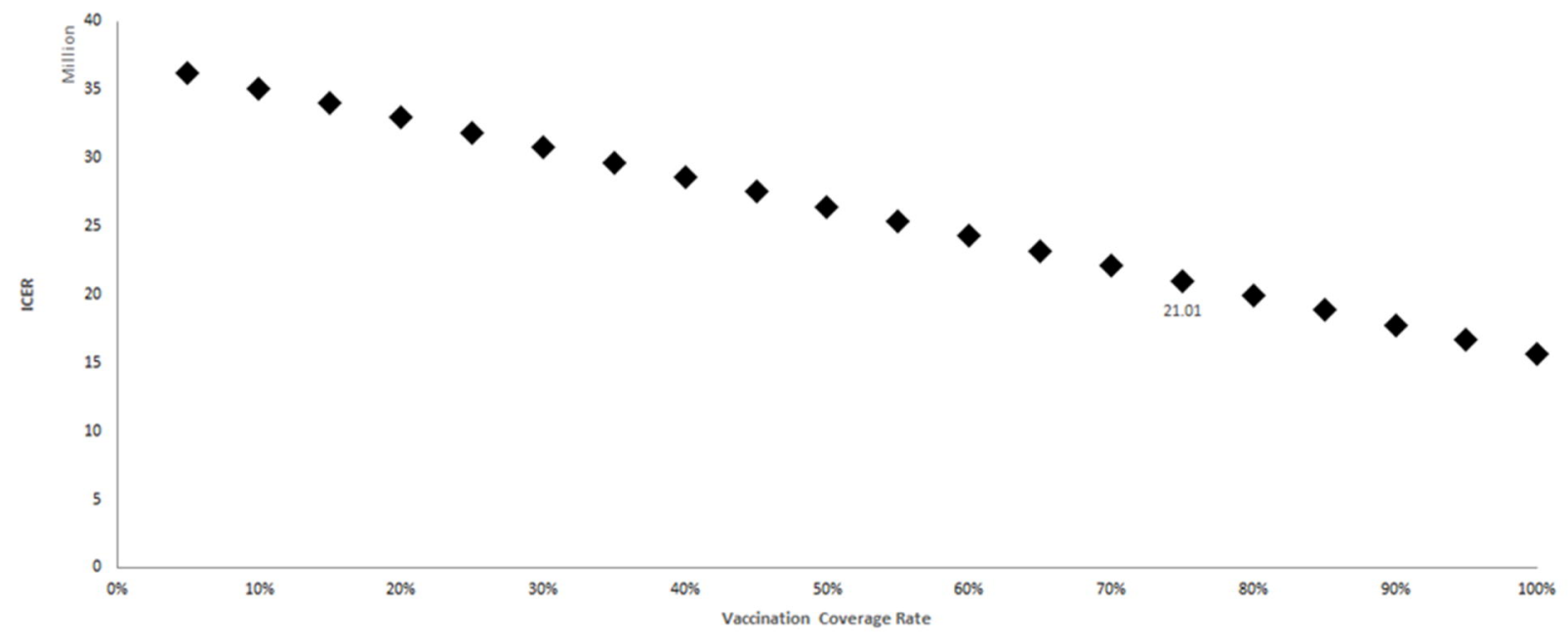




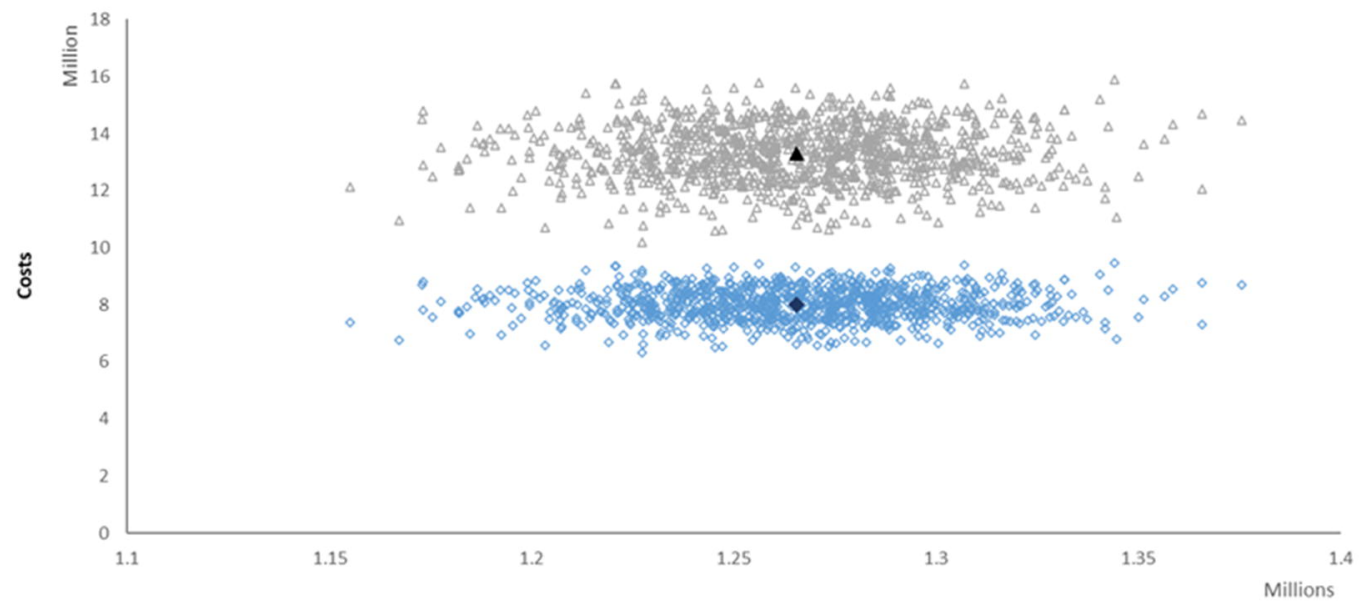

- Base Case TIV 